\title{
ESTUDO DA EFICIÊNCIA DE DIFERENTES FONTES DE FOSFATO NA CULTURA DO SORGO
}

\author{
Izabel Maria Almeida Lima ${ }^{1}$, Boanerges Freire de Aquino², Bruno Lucio Meneses \\ Nascimento $^{3}$ \\ 1 Doutoranda em Ciência do Solo na Universidade Federal do Ceará, Fortaleza (CE). E-mail: \\ izabel.agro@yahoo.com.br \\ ${ }^{2}$ Professor Titular PhD. do Departamento de Ciências do Solo da Universidade Federal do Ceará, Fortaleza \\ (CE). E-mail: aquino@ufc.br \\ ${ }^{3}$ Doutorando em Engenharia Civíl - Saneamento Ambiental na Universidade Federal do Ceará, Fortaleza \\ (CE). E-mail: brunonoimpma@ hotmail.com
}

RESUMO: Os baixos teores de fósforo disponíveis nos solos tropicais, em geral, requerem permanentes pesquisas com relação à eficiência das aplicações de diferentes fontes de adubos fosfatados nas culturas cujo objetivo é elevar os teores desse nutriente no solo. Este trabalho teve como objetivo, estudar as respostas do sorgo às aplicações de diferentes doses e fontes de fósforo a produção de matéria seca, além de avaliar a eficiência agronômica de diferentes fontes. Foi utilizado o delineamento em blocos inteiramente casualizados, em esquema fatorial $4 \times 4$ (doses e fontes de $\mathrm{P}$, respectivamente), sendo os tratamentos formado por quatro doses de fósforo (dose $1=0$, dose $2=100$, dose $3=200$ e dose $4=300 \mathrm{mg}$ de $\mathrm{P}$ $\mathrm{kg}^{-1}$ solo) e quatro fontes de P (Fosfato Industrial Superfosfato Triplo - ST, Fosfato Natural da Bahia - FNB, Fosfato Natural do Tocantins - FNT e Fosfato Natural Reativo - Gafsa FNR), com 4 repetições, totalizando 64 unidades experimentais. Foram avaliadas as seguintes variáveis: altura de planta, diâmetro do caule, produção de matéria seca da parte aérea e os níveis foliares de macronutrientes e micronutrientes. As variáveis foram submetidas à análise de variância (ANOVA), análise de regressão de acordo com fontes e doses crescentes de fósforo, as médias dessas variáveis foram avaliadas pelo teste de Tukey a 5\% de probabilidade. O superfosfato triplo (ST) e fontes FNB foram responsáveis pelas respostas significativas mais elevados das variáveis biométricas estudadas, enquanto FNT teve a menor resposta.

Palavras-chave: Eficiência de adubo. Fósforo. Sorghum bicolor.

\section{STUDY OF THE EFFICIENCY OF DIFFERENT PHOSPHATE SOURCES IN SORGHUM GROWN}

\begin{abstract}
The low available of phosphorus in tropical soils generally require ongoing research regarding the efficiency and the application of different sources of phosphate fertilizers on crops. This work aimed to study the responses of sorghum applications of different levels and sources of phosphorus in relation to growth, yield and nutrient removal, and to evaluate the agronomic efficiency of different sources. The experiment was conducted in a greenhouse at the Federal University of Ceará. We used soil from Chapada do Apodi-CE Cambisol. The present study used a randomized complete block design in a
\end{abstract}

Cultura Agronômica, Ilha Solteira, v.25, n.1, p.25-38, 2016 
4x4 factorial scheme (doses and sources of $\mathrm{P}$, respectively), with treatments consist of four doses of phosphorus (dose $1=0$, dose $2=100$, dose $3=200$ and dose $4=300 \mathrm{mg} \mathrm{P} \mathrm{kg}{ }^{-1}$ soil) and four sources of $\mathrm{P}$ (Phosphate Industrial Triple superphosphate - ST, Natural Phosphate of Bahia - FNB, Natural Phosphate of Tocantins - FNT and Natural Reactive phosphate - Gafsa - FNR), with 4 replicates, totalizing 64 experimental units. We evaluated the following variables: plant height, stem diameter, dry matter production of shoot and leaf levels of macronutrients and micronutrients. The variables were subjected to analysis of variance (ANOVA), regression analysis according to sources and increasing doses of phosphorus, the averages of these variables were evaluated by the Tukey test at 5\% probability. The triple superphosphate (ST) and FNB sources were responsible for the higher significant responses of biometric variables studied, whereas FNT had the lowest response.

Key words: Fertilizer efficiency. Phosphorus. Sorghum bicolor.

\section{INTRODUÇÃO}

O sorgo pertence à família Poaceae, gênero Sorghum e a espécie cultivada é Sorghum bicolor (L.) Moench. É uma cultura que apresenta alto potencial para alimentação de animais, podendo ser empregado nas regiões semiáridas, por ser resistente à seca e altas temperaturas, sendo diferencial em relação às outras culturas e com isso tem sua importância em regiões onde não dispõem de irrigação artificial (OLIVEIRA et al., 2002).

A produtividade de sorgo no Brasil compreende a faixa de aproximadamente $2768 \mathrm{~kg}$ $\mathrm{ha}^{-1}$ em média (CONAB, 2014). Este fato para o Brasil é estrategicamente importante, pois ter uma área ocupada com sorgo, para a garantia do abastecimento de grãos, será de fundamental importância. A produção brasileira de grãos depende quase que exclusivamente da precipitação pluviométrica. Em anos com a ocorrência de condições desfavoráveis, normalmente há déficit na produção de grãos e o sorgo, sendo uma cultura de vocação para cultivo em condições adversas de clima e solo, poderia reduzir o impacto desse fator no abastecimento de grãos (MAGALHÃES et al., 2000).

Os nutrientes têm funções essenciais e específicas no metabolismo das plantas. Dessa forma, quando um dos nutrientes essenciais não está presente em quantidades satisfatórias ou em condições que o tornem pouco disponível, a sua deficiência nas células promove alterações no seu metabolismo (TAIZ; ZEIGER, 2004). O P é um dos nutrientes que mais limitam a produtividade das culturas nos solos de carga variável que predominam nas regiões tropicais e subtropicais, caso dos solos brasileiros (MEURER, 2006), porém é o nutriente mais utilizado como fertilizante, sendo, portanto, este elemento discutido de modo mais abrangente e com mais detalhes.

As principais fontes de Fósforo (P) comercializadas no Brasil são os fosfatos acidulados, os fosfatos naturais e os termofosfatos. No entanto na agricultura brasileira mais de $90 \%$ dos fosfatos utilizados são os acidulados (GOEDERT et al., 1990). Lopes (1989) afirma que a adubação fosfatada além de promover a formação e o crescimento prematuro 
de raízes, também melhora a eficiência no uso da água, e quando se encontra em alto nível no solo, ajuda a manter a absorção deste pelas mesmas.

Vance et al. (2003) estimam que as reservas de fósforo no mundo economicamente viáveis para exploração podem ser suficientes até 2050, indicando a necessidade de estudar a eficiência de fontes alternativas de $\mathrm{P}$ para as culturas. A eficiência agronômica dos adubos fosfatados pode ser avaliada por diversos índices, dentre os quais se destacam a solubilidade em ácido cítrico a 2\%, o índice de eficiência agronômica (IEA) e o equivalente em superfosfato triplo (EqST), cuja eficiência agronômica é aferida por meio de índices estabelecidos pela comparação com um fosfato padrão, em geral o superfosfato triplo. Diversos trabalhos evidenciam baixa eficiência agronômica de alguns fosfatos naturais (LEÓN et al., 1986; KORNDÖRFER et al., 1999); e pode ser afetada pelas fontes de fosfato, propriedades do solo, modos de aplicação e espécies vegetais (CHIEN; MENON, 1995).

A deficiência de informações relativas à nutrição e adubação fosfatada do sorgo forrageiro para as condições tropicais brasileiras justifica a realização de estudos e este tem como principal objetivo avaliar a eficiência de diferentes fontes de fósforo e quantificar teores de $\mathrm{P}$ na cultura do sorgo.

\section{MATERIAL E MÉTODOS}

O experimento foi realizado em casa de vegetação, do Departamento de Ciências do Solo, do Centro de Ciências Agrárias, da Universidade Federal do Ceará, Campus do Pici em Fortaleza, Ceará, Brasil.

O solo usado no experimento é classificado como Cambissolo Háplico Eutrófico segundo o Sistema Brasileiro de Classificação de Solos (EMBRAPA, 2006), retirado da Chapada do Apodi localizada no município de Limoeiro do Norte, estado do Ceará, a 198 km de distância da capital Fortaleza.

As características físicas e químicas do solo desta região se encontram na Tabela 1.

Foi retirada a camada superior do solo $(0-25 \mathrm{~cm})$ em uma área de mata nativa, com remoção da cobertura vegetal; as amostras foram colocadas em sacos plásticos com capacidade de aproximadamente $60 \mathrm{~kg}$, sendo transportados para a casa de vegetação do Departamento de Ciência do Solo da Universidade Federal do Ceará. Posteriormente, as amostras foram secas ao ar, destorroadas, passadas em peneira de malha de $2 \mathrm{~mm}$ de abertura.

Para a condução do experimento foram analisadas quatro fontes de fósforo, sendo, uma Fonte Industrial (Superfosfato Triplo - ST) e três Fontes Naturais (Fosfato Natural de Bahia - FNB, Fosfato Natural do Tocantins - FNT e Fosfato Natural Reativo - FNR).

Foram realizadas análises para determinação do teor de fósforo (percentagem) de cada uma das fontes acima relacionadas e usadas no experimento. $\mathrm{O}$ fósforo total presente nos fosfatos naturais foi analisado de acordo com o método colorimétrico do ácido 
molibdovanadofosfórico e a determinação do teor de fósforo total no superfosfato triplo foi determinado em espectrofotômetro a $660 \mathrm{~nm}$ (EMBRAPA, 1997). A Tabela 2 apresenta os teores de fósforo encontrados nas diferentes fontes.

Tabela 1. Características físico-químicas do Cambissolo usado no experimento.

\begin{tabular}{|c|c|}
\hline $\mathrm{pH}\left(\mathrm{H}_{2} \mathrm{O}\right)$ & 6,8 \\
\hline M.O $\left(\mathrm{g} \mathrm{kg}^{-1}\right)$ & 20,8 \\
\hline $\mathrm{C}\left(\mathrm{g} \mathrm{kg}^{-1}\right)$ & 12,0 \\
\hline $\mathrm{N}\left(\mathrm{g} \mathrm{kg}^{-1}\right)$ & 1,1 \\
\hline $\mathrm{CE}\left(\mathrm{dS} \mathrm{m} \mathrm{m}^{-1}\right)$ & 0,3 \\
\hline $\mathrm{P}\left(\mathrm{mg} \mathrm{kg}^{-1}\right)$ & 1,0 \\
\hline $\mathrm{Na}^{+}\left(\mathrm{cmol}_{\mathrm{c}} \mathrm{kg}^{-1}\right)$ & 0,1 \\
\hline $\mathrm{K}^{+}\left(\mathrm{cmol}_{\mathrm{c}} \mathrm{kg}^{-1}\right)$ & 0,5 \\
\hline $\mathrm{Ca}^{2+}\left(\mathrm{cmol}_{\mathrm{c}} \mathrm{kg}^{-1}\right)$ & 4,4 \\
\hline $\mathrm{Mg}^{2+}\left(\mathrm{cmol}_{\mathrm{c}} \mathrm{kg}^{-1}\right)$ & 1,8 \\
\hline $\mathrm{H}^{+}+\mathrm{Al}^{3+}\left(\mathrm{cmol}_{\mathrm{c}} \mathrm{kg}^{-1}\right)$ & 1,9 \\
\hline $\mathrm{Al}^{3+}\left(\mathrm{cmol}_{\mathrm{c}} \mathrm{kg}^{-1}\right)$ & 0,1 \\
\hline $\mathrm{SB}\left(\mathrm{cmol}_{\mathrm{c}} \mathrm{kg}^{-1}\right)$ & 6,8 \\
\hline $\mathrm{T}\left(\mathrm{cmol}_{\mathrm{c}} \mathrm{kg}^{-1}\right)$ & 8,8 \\
\hline $\mathrm{V}(\%)$ & 77 \\
\hline $\mathrm{m}(\%)$ & 1 \\
\hline PST & 1 \\
\hline Areia Grossa $\left(\mathrm{g} \mathrm{kg}^{-1}\right)$ & 457 \\
\hline Silte $\left(\mathrm{g} \mathrm{kg}^{-1}\right)$ & 131 \\
\hline Argila $\left(\mathrm{g} \mathrm{kg}^{-1}\right)$ & 240 \\
\hline Argila natural $\left(\mathrm{g} \mathrm{kg}^{-1}\right)$ & 110 \\
\hline Densidade Global $\left(\mathrm{g} \mathrm{cm}^{-3}\right)$ & 1,43 \\
\hline Classificação Textural & Franco Argilo Arenos \\
\hline
\end{tabular}

Tabela 2. Teor de $\mathrm{P}$ e $\mathrm{P}_{2} \mathrm{O}_{5}$ total encontrado nos fosfatos naturais e no superfosfato triplo.

\begin{tabular}{ccc}
\hline Fonte & Fósforo $(\%)$ & $\mathrm{P}_{2} \mathrm{O}_{5}(\%)$ \\
SFT & 18,0 & 41 \\
FNB & 9,5 & 21 \\
FNT & 6,1 & 15 \\
FNR & 10,9 & 25 \\
\hline
\end{tabular}

Após a coleta das amostras de solos e análises dos teores de fósforo das fontes, foi realizado o preenchimento dos vasos com $4 \mathrm{~kg}$ de solo para o cultivo do sorgo. O plantio das sementes certificadas do sorgo fornecidas pela Secretaria do Desenvolvimento Agrário DAS, sede em Fortaleza-CE, foi realizado em novembro de 2012.

Para o delineamento experimental foram utilizadas quatro doses de Fósforo $(\mathrm{P}), 0$, 100, 200 e $300 \mathrm{mg}$ de $\mathrm{P} \mathrm{kg}^{-1}$, com quatro repetições. O delineamento experimental utilizado foi o de blocos inteiramente casualizados dispostos em um esquema fatorial 4.4.4 (doses de 
$\mathrm{P}$, fontes de $\mathrm{P}$ e repetições, respectivamente). Desta forma, o experimento constou 16 tratamentos totalizando 64 unidades experimentais.

Nos vasos com $4 \mathrm{~kg}$ de solo, foram plantadas oito sementes por vaso. Após sete dias do plantio foi realizado o desbaste, deixando-se três plantas por vaso. A adubação básica foi constituída de Sulfato de Amônio - $\left(\mathrm{NH}_{4}\right)_{2} \mathrm{SO}_{4}$, (1000 mg de N/ vaso), Cloreto de Potássio $\mathrm{KCl}$ (250 mg de K vaso ${ }^{-1}$ ) e Micronutrientes: Sulfato Manganoso Hidratado $-\mathrm{MnSO}_{4} \cdot \mathrm{H}_{2} \mathrm{O}$ (25 mg de $\mathrm{Mn}_{\text {vaso }}{ }^{-1}$ ), Fe-EDTA (12 mg de Fe vaso ${ }^{-1}$ ), Sulfato Cúprico Hidratado $-\mathrm{CuSO}_{4}$ $5 \mathrm{H}_{2} \mathrm{O}$ (5 mg de $\mathrm{Cu}$ vaso $\left.^{-1}\right)$, Tetraborato de Sódio Hidratado $-\mathrm{Na}_{2} \mathrm{~B}_{4} \mathrm{O}_{7}, 10 \mathrm{H}_{2} \mathrm{O}(8 \mathrm{mg}$ de $\mathrm{B}$ vaso $\left.^{-1}\right)$, Molibdato de Amônio - $\left(\mathrm{NH}_{4}\right)_{6} \mathrm{Mo}_{7} \mathrm{O}_{24} .4 \mathrm{H}_{2} \mathrm{O}\left(1 \mathrm{mg}\right.$ de Mo vaso $\left.{ }^{-1}\right)$ e Sulfato de Zinco Hidratado - $\mathrm{ZnSO}_{4} 7 \mathrm{H}_{2} \mathrm{O}$ (16 mg de $\mathrm{Zn}$ vaso $\left.^{-1}\right)$.

A irrigação dos vasos foi realizada diariamente com base no peso dos vasos, procurando-se manter o teor de umidade constante. Por fim a colheita do material para análises ocorreu aos 45 dias após a emergência das sementes.

Ao final do experimento, após os 45 dias depois da emergência, as plantas foram coletadas para análises laboratoriais. As partes aéreas foram acondicionadas em sacos de papel e posteriormente secadas em estufa de circulação forçada de ar $\left(65^{\circ} \mathrm{C}\right)$, até atingir massa constante em $48 \mathrm{~h}$. Logo em seguida, o material vegetal foi pesado para obtenção da produção da matéria seca da parte aérea - PMSPA (em g/vaso).

Depois da determinação da PMSPA, foi efetuada a moagem para digestão e realização da determinação do teor de fósforo no tecido vegetal. Para as determinações dos nutrientes acima relacionados, foi utilizada a digestão úmida com solução nitro-perclórica (3:1) de acordo com metodologia proposta por Malavolta et al. (1997) e Embrapa (1997). A determinação do fósforo foi realizada por espectrofotometria com azul-de-molibdênio e a leitura foi realizada no espectrofotômetro a $660 \mathrm{~nm}$.

O índice equivalente supertriplo foi utilizado para relacionar percentualmente a produção de matéria seca na parte aérea obtida utilizando uma dose de um fertilizante-teste de fósforo e a produção obtida com a mesma dose na forma de superfosfato triplo de acordo com a equação abaixo:

$$
\text { Índice Eq. Supertriplo }(\%)=\left(\mathrm{P}_{1} / \mathrm{P}_{2}\right) \times 100
$$

\section{Onde:}

$\mathrm{P}_{1}=$ Produção da massa seca da parte aérea (MSPA) com certa dose de fosfato natural;

$\mathrm{P}_{2}=$ Produção da massa seca da parte aérea (MSPA) com certa dose do superfosfato triplo.

O índice de eficiência agronômica (IEA) foi calculado com os valores de produção de massa seca da parte aérea (PMSPA), conforme equação abaixo descrita por Chien e Hammond (1978) e utilizada por Moreira, Malavolta e Morais (2002) e Franzini et al. (2009).

$$
\left.\operatorname{IEA}(\%)=\left[\left(\mathrm{M}_{2}-\mathrm{M}_{1}\right)\right] /\left(\mathrm{M}_{3}-\mathrm{M}_{1}\right)\right] \text { x } 100
$$

\section{Onde:}

$\mathrm{M}_{1}=$ Produção da MSPA na testemunha (sem P);

Cultura Agronômica, Ilha Solteira, v.25, n.1, p.25-38, 2016 
$\mathrm{M}_{2}=$ Produção da MSPA com a fonte testada nas diferentes doses;

$\mathrm{M}_{3}=$ Produção da MSPA com a fonte de referência (SFT) nas diferentes doses de P.

As variáveis estudadas foram submetidas à análise de variância (ANOVA), relacionando as fontes, doses e interação de fontes e doses. Sendo as médias avaliadas pelo teste de Tukey, a 1 e a $5 \%$ de probabilidade, utilizando o programa ASSISTAT Versão 7.6 Beta (SILVA; AZEVEDO, 2002). Além disso, foram realizadas análises de regressão com as variáveis e as doses de fósforo das fontes.

\section{RESULTADOS E DISCUSSÃO}

Analisando-se a Tabela 3, pode ser notado que, com o aumento da adubação fosfatada houve incremento na matéria seca em todos os tratamentos. $\mathrm{O}$ fator solubilidade das fontes foi fator determinante para os aumentos dos valores de MSPA. Pesquisas demonstram influência nos teores e acúmulo de $\mathrm{P}$ no solo uma vez que, de maneira geral, as fontes mais disponíveis (como as fontes usadas no presente estudo) proporcionaram maiores MSPA e, consequentemente, maiores acúmulos de $\mathrm{P}$ na planta.

Tabela 3. Produção de Matéria Seca da Parte Aérea-PMSPA (g vaso-1) das plantas de sorgo em função de diferentes doses e fontes de fósforo.

\begin{tabular}{ccccc}
\hline \multicolumn{5}{c}{$\begin{array}{c}\text { Doses } \\
\left(\mathbf{m g ~ P ~ k g}^{-1}\right)\end{array}$} \\
\hline Fontes & $\mathbf{0}$ & $\mathbf{1 0 0}$ & $\mathbf{2 0 0}$ & $\mathbf{3 0 0}$ \\
SFT & $0,78 \mathrm{a}$ & $3,39 \mathrm{~b}$ & $4,01 \mathrm{~b}$ & $5,04 \mathrm{a}$ \\
FNB & $0,78 \mathrm{a}$ & $3,72 \mathrm{a}$ & $3,91 \mathrm{c}$ & $4,74 \mathrm{~b}$ \\
FNT & $0,78 \mathrm{a}$ & $2,07 \mathrm{~d}$ & $2,20 \mathrm{~d}$ & $2,40 \mathrm{~d}$ \\
FNR & $0,78 \mathrm{a}$ & $3,25 \mathrm{c}$ & $4,25 \mathrm{a}$ & $4,35 \mathrm{c}$ \\
\hline CV\% & \multicolumn{5}{c}{1,07} \\
\hline
\end{tabular}

Médias seguidas por letras distintas dentro de cada dose diferem entre si pelo teste de Tukey a $5 \%$ de probabilidade; C.V= Coeficiente de Variação; SFT= Superfosfato triplo; FNB= Fosfato Natural da Bahia; FNT= Fosfato Natural do Tocantins; FNR= Fosfato Natural Reativo.

$\mathrm{Na}$ dose $100 \mathrm{mg} \mathrm{kg}^{-1}$ solo, houve diferença estatística entre todas as fontes, sendo o FNB, a fonte que proporcionou o maior valor $(3,72 \mathrm{~g})$, seguida do Superfosfato triplo $(3,39$ g), FNR (3,25 g) e o FNT com o menor valor (2,07 g). Na dose $200 \mathrm{mg} \mathrm{kg}^{-1}$ solo, houve diferença estatística entre todas as fontes, sendo o FNR que teve maior valor $(4,25 \mathrm{~g})$, seguido do Superfosfato triplo $(4,01)$, FNB $(3,91 \mathrm{~g})$ e o FNT com o menor valor $(2,20 \mathrm{~g})$; na dose $300 \mathrm{mg} \mathrm{kg}^{-1}$ solo, houve diferença estatística entre todas as fontes, sendo a fonte Superfosfato triplo a que proporcionou o maior valor $(5,04 \mathrm{~g})$, seguida FNB $(4,74 \mathrm{~g})$, FNR $(4,35 \mathrm{~g})$ e o FNT com o menor valor $(2,40 \mathrm{~g})$.

Dentre os fosfatos naturais, notou-se que os maiores valores de PMSPA foram obtidos com a fonte $\mathrm{FNB}$, na dose $300 \mathrm{mg} \mathrm{kg}^{-1}$ solo, cujo aumento percentual em relação a dose 0 foi de 507\%. A fonte FNT, em todas as doses avaliadas, foi a que causou a menor produção de matéria seca. Portanto, nessas condições de estudo o ideal seria utilizar $300 \mathrm{mg}$ de P,

Cultura Agronômica, Ilha Solteira, v.25, n.1, p.25-38, 2016 
tendo como fonte o ST para obtenção de máxima produção, sendo o ST superior ao $1^{\circ}$ tratamento (dose 0) em 4,26 g, um aumento equivalente a 546\%.

Estes resultados corroboram com aqueles obtidos por Leite (2006), o qual também verificou incremento na produção de matéria seca de sorgo em função da adubação fosfatada.

Avaliando a interação doses e fontes para esta variável através da regressão (Figura 1), cujos dados ajustaram ao modelo quadrático, pode-se notar que a máxima produção de matéria seca parte aérea do sorgo foi atingida com as doses teóricas de $300 \mathrm{mg} \mathrm{kg}^{-1}$ solo do superfosfato triplo, $263 \mathrm{mg} \mathrm{kg}^{-1}$ solo do FNB, $243 \mathrm{mg} \mathrm{kg}^{-1}$ solo do FNT e $240 \mathrm{mg} \mathrm{kg}^{-1}$ solo do FNB. Sendo que a maior produção de matéria seca foi conseguida através do Superfosfato triplo(5,04g).

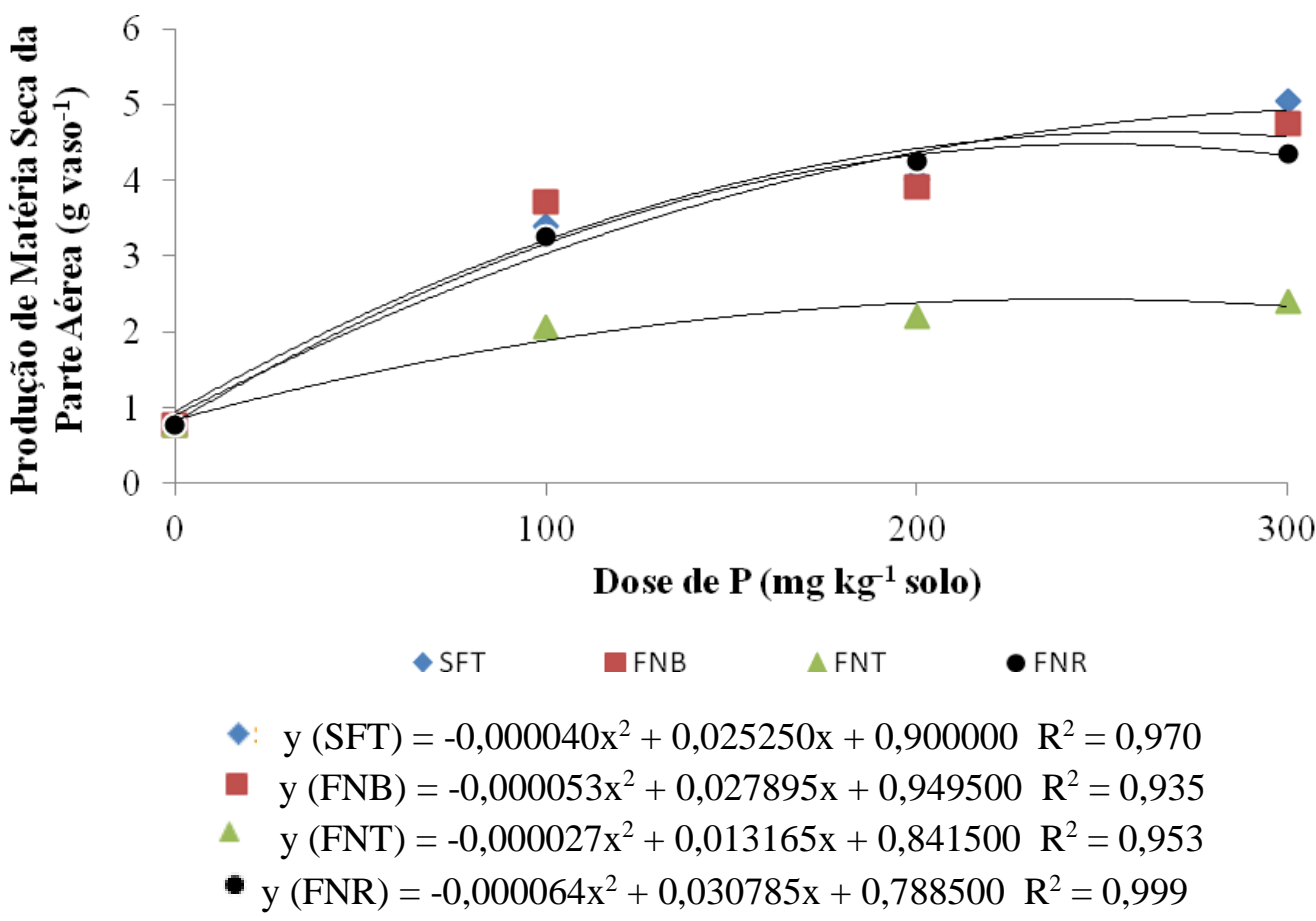

Figura 1. Efeitos das doses de fósforo de diferentes fontes sobre a produção de matéria seca da parte aérea - PMSPA das plantas de sorgo.

Hoffmann et al. (1995) também obtiveram os mesmos resultados trabalhando com o capim braquiária (Brachiaria decumbens Stapf), verificando um aumento da produção de MS com adubação de P. E Segatelli (2004), também verificou que a adubação fosfatada recomendada para a cultura do sorgo, a produção de matéria seca foi incrementada.

Comportamento similar foi verificado em outros estudos, como o de Sarmento et al. (2001) ao avaliarem a produção de massa seca de alfafa, onde verificaram que as maiores produções de massa seca foram alcançadas, quando se utilizou a fonte de $\mathrm{P}$ de maior solubilidade (ST), quando comparada com o fosfato de Gafsa; no trabalho de Corrêa et al. (2005) foi constatada a superioridade do ST em relação ao fosfato de Gafsa na produção de massa seca da parte aérea do milho.

Os resultados do fósforo absorvidos pelo sorgo são apresentados na Tabela 4.

Cultura Agronômica, Ilha Solteira, v.25, n.1, p.25-38, 2016 
Tabela 4. Quantidades de fósforo $\left(\mathrm{mg} \mathrm{vaso}^{-1}\right)$ encontradas na parte aérea do sorgo em função de diferentes doses e fontes de fósforo.

\begin{tabular}{c|lcrc}
\hline \multicolumn{5}{c}{$\begin{array}{c}\text { Doses } \\
\left(\mathbf{m g ~ P ~ k g}^{-1}\right)\end{array}$} \\
\hline Fontes & $\mathbf{0}$ & $\mathbf{1 0 0}$ & $\mathbf{2 0 0}$ & $\mathbf{3 0 0}$ \\
SFT & $0,17 \mathrm{a}$ & $10,16 \mathrm{a}$ & $16,64 \mathrm{a}$ & $18,74 \mathrm{a}$ \\
FNB & $0,17 \mathrm{a}$ & $7,10 \mathrm{~b}$ & $8,42 \mathrm{~b}$ & $8,77 \mathrm{~b}$ \\
FNT & $0,17 \mathrm{a}$ & $2,04 \mathrm{~d}$ & $2,54 \mathrm{~d}$ & $3,33 \mathrm{~d}$ \\
FNR & $0,17 \mathrm{a}$ & $5,03 \mathrm{c}$ & $5,27 \mathrm{c}$ & $7,29 \mathrm{c}$ \\
\hline CV\% & \multicolumn{5}{c}{}
\end{tabular}

Médias seguidas por letras distintas dentro de cada dose diferem entre si pelo teste de Tukey a 5\% de probabilidade; $\mathrm{C} . \mathrm{V}=$ Coeficiente de Variação; SFT= Superfosfato triplo; FNB= Fosfato Natural da Bahia; FNT= Fosfato Natural do Tocantins; FNR= Fosfato Natural Reativo.

Quando comparadas com as absorções de $\mathrm{P}$ pelo tratamento na dose 0 , as maiores remoções de $\mathrm{P}$ pelo sorgo, e estatisticamente significativas (pelo teste de tukey, a $5 \%$ de probabilidade), foram obtidas com as doses da fonte ST. Dentre as fontes, o FNT foi a fonte que proporcionou as menores taxas de remoções de $\mathrm{P}$ pelo sorgo.

A absorção de P na parte aérea das plantas apresentou comportamento quadrático para todas as fontes usadas (Figura 2), mostrando um acréscimo no teor de P com o aumento da dose, sendo que as diferenças entre as fontes se tornam evidentes pelas tendências das curvas. Este resultado indica que para a obtenção de maiores remoções de $\mathrm{P}$ pela planta ocorreram nas doses teóricas de 300; 231; 327; e $300 \mathrm{mg} \mathrm{P} \mathrm{kg}^{-1}$ solo, cujas as fontes foram Superfosfato triplo, FNB, FNT e FNR, respectivamente.

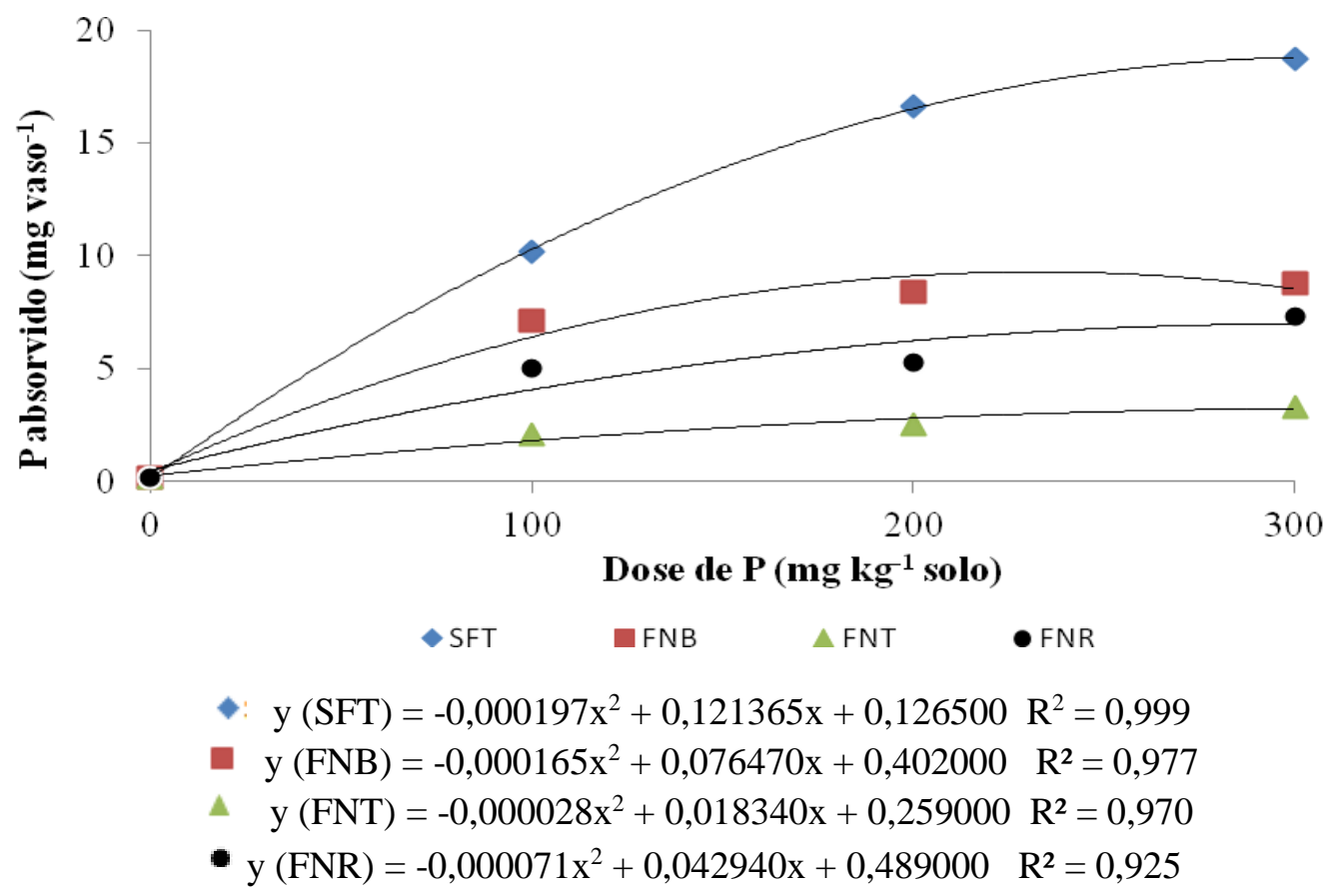

Figura 2. Quantidades de fósforo na parte aérea de plantas de sorgo (mg vaso $\left.{ }^{-1}\right)$ em função das crescente doses e diferentes fontes de $\mathrm{P}$. 
De acordo com a figura 2, fica demonstrado que no ST, na dose $300 \mathrm{mg} \mathrm{kg}^{-1}$ solo, foi a fonte responsável pela maior absorção de fósforo $\left(18,74 \mathrm{mg} \mathrm{P}\right.$ vaso $\left.^{-1}\right)$. $\mathrm{E}$ entre as fontes naturais, o FNB, foi o que proporcionou a maior absorção de fósforo também na dose 300 $\mathrm{mg} \mathrm{kg}^{-1}$ solo $\left(8,77 \mathrm{mg} \mathrm{P}\right.$ vaso $\left.^{-1}\right)$.

Gill et al. (1992), trabalhando com milho em diferentes doses de $\mathrm{P}$, observaram que as diferentes quantidades de fósforo removido pelo o sorgo é explicada pela capacidade que cada fonte tem de liberar fósforo para o solo, ou seja, à medida que a concentração de $\mathrm{P}$ no meio aumentou, aumentou a proporção de $\mathrm{P}$ acumulada na parte aérea da planta estudada.

Costa et al. (2008), estudando aplicações de fontes de $\mathrm{P}$ no solo nas formas de superfosfato triplo, fosfato de Arad, fosfato natural de Araxá e, uma mistura superfosfato triplo + fosfato de Arad na relação (1:1), cultivado com Brachiaria brizantha, relataram que o teor de $\mathrm{P}$ na parte aérea aumentava significativamente ao se utilizar as fontes de $\mathrm{P}$ de maior solubilidade.

Os percentuais do índice de equivalência de supertriplo para as fontes avaliadas estão na Tabela 5. Dentre os fosfatos naturais avaliadas na dose $100 \mathrm{mg} \mathrm{kg}^{-1}$ solo, as fontes FNB e FNR foram as fontes que apresentaram a equivalência mais próxima da fonte ST. Observouse também que a fonte FNT foi a que obteve o menor índice de equivalente supertriplo, o que indica baixa eficiência dessa fonte em aumentar a matéria seca produzida pelo sorgo. Pode-se observar também que todas as fontes avaliadas diferem estatisticamente entre si, pelo teste de Tukey a 5\%, nas doses 100, 200 e $300 \mathrm{mg} \mathrm{kg}^{-1}$ solo.

Tabela 5. Índice de Equivalente Supertriplo (\%) das fontes de fósforo utilizadas no cultivo do sorgo.

\begin{tabular}{|c|c|c|c|c|}
\hline \multicolumn{5}{|c|}{$\begin{array}{c}\text { Doses } \\
\left(\mathrm{mg} \mathrm{P} \mathrm{kg}^{-1}\right)\end{array}$} \\
\hline Fontes & $\mathbf{0}$ & 100 & 200 & 300 \\
\hline SFT & $0,78 \mathrm{a}$ & $100,0 \mathrm{a}$ & $100,0 \mathrm{a}$ & $100,0 \mathrm{a}$ \\
\hline FNB & $0,78 \mathrm{a}$ & $96,16 \mathrm{~b}$ & $92,76 \mathrm{c}$ & $94,09 \mathrm{~b}$ \\
\hline FNT & $0,78 \mathrm{a}$ & $61,27 \mathrm{c}$ & $55,04 \mathrm{~d}$ & $47,76 \mathrm{~d}$ \\
\hline FNR & $0,78 \mathrm{a}$ & $96,09 \mathrm{~b}$ & $98,18 \mathrm{~b}$ & $84,37 \mathrm{c}$ \\
\hline \multicolumn{2}{|l|}{ CV\% } & 1,10 & & \\
\hline
\end{tabular}

Médias seguidas por letras distintas dentro de cada dose diferem entre si pelo teste de Tukey a 5\% de probabilidade; $\mathrm{C} . \mathrm{V}=$ Coeficiente de Variação; SFT= Superfosfato triplo; FNB= Fosfato Natural da Bahia; FNT= Fosfato Natural do Tocantins; FNR= Fosfato Natural Reativo.

Segundo Novais e Smyth (1999), quando as apatitas brasileiras de baixa reatividade são comparadas com fosfatos naturais de maior reatividade, como o de Gafsa, Carolina do Norte e fosfatos industrializados, observa-se que as apatitas brasileiras geralmente apresentam comportamento insatisfatório em fornecer fósforo para as culturas. Essa evidência foi comprovada no presente trabalho quando as fontes FNR e FNT são comparadas. Santos e Kliemann (2005) chegaram à conclusão de que os fosfatos de Arad e Araxá produziram 24 e 53\% a menos de matéria seca em relação a matéria seca obtida com a fonte ST. Ao analisar a média dos percentuais de equivalência das 4 fontes avaliadas no 
presente trabalho, verificou-se que o índice equivalente supertriplo seguiu a seguinte ordem decrescente: $\mathrm{ST}>\mathrm{FNB}>\mathrm{FNR}>\mathrm{FNT}$. Sendo assim, no presente estudo, a fonte FNB e o FNR, em todas as doses avaliadas, são classificadas como fontes de alta solubilidade.

A análise de regressão para o índice de equivalente supertriplo dos fosfatos naturais em função de doses crescentes de fósforo está representada na Figura 3. Os percentuais de equivalência das fontes FNB, FNT e FNR melhor se adequaram ao modelo quadrático de regressão, sendo que a máxima equivalência pode ser obtida com as doses 214 do FNB, 300 do FNT e $196 \mathrm{mg} \mathrm{kg}^{-1}$ de P no solo do FNR.

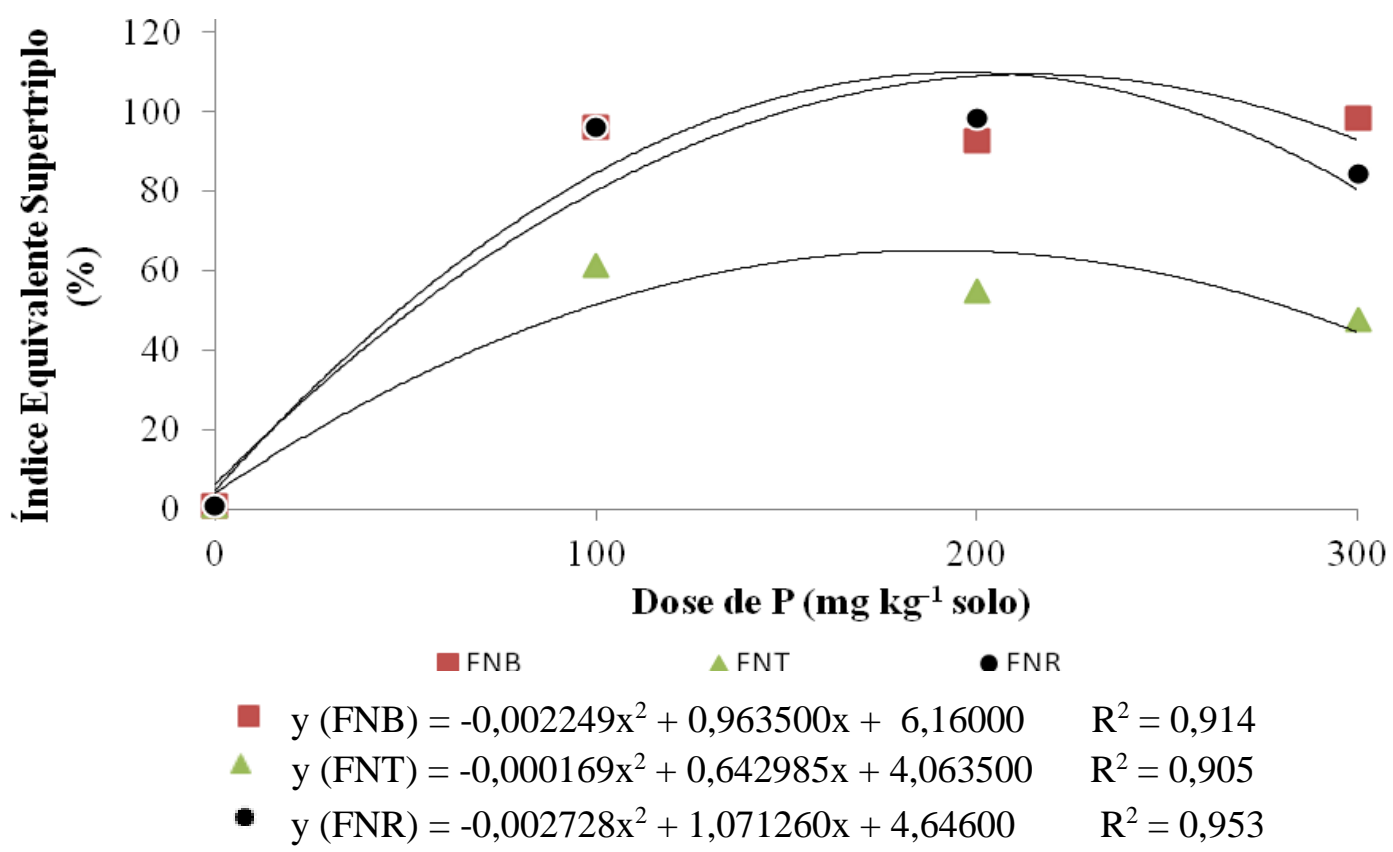

Figura 3. Análise de regressão do Índice de Equivalente Supertriplo (\%) dos fosfatos naturais em função de doses crescentes de fósforo.

Na Tabela 6, observa-se que ocorreram efeitos das doses, das fontes e interação desses fatores nos valores do IEA. Ficou demonstrado que o ST foi a fonte de fósforo significativamente de maior eficiência. Dentre os fosfatos naturais avaliados, o FNR, na dose $200 \mathrm{mg} \mathrm{kg}^{-1}$ solo, foi a fonte que apresentou eficiência similar ao da fonte ST.

Os fosfatos naturais avaliados apresentaram eficiências agronômicas estatisticamente diferentes, entre si, na dose $100 \mathrm{mg} \mathrm{kg}^{-1}$ solo, sendo que as fontes FNB e FNR apresentaram eficiências agronômicas estatisticamente iguais. A fonte FNT apresentou o menor valor de IEA.

Analisando-se a média dos Índices de Eficiência Agronômica das fontes, verificou-se que esse índice seguiu a seguinte ordem decrescente: ST> FNB> FNR> FNT. Goedert e Lobato (1984); e Rosand e Santana (1986) trabalhando com sorgo, observaram que o IEA do fosfato de Gafsa foi semelhante, ou superior, ao do ST.

Cultura Agronômica, Ilha Solteira, v.25, n.1, p.25-38, 2016 
Tabela 6. Índice de Eficiência Agronômica (\%) das fontes de fósforo utilizadas no cultivo do sorgo.

\begin{tabular}{c|cccc}
\hline \multicolumn{5}{c}{$\begin{array}{c}\text { Doses } \\
\left(\mathbf{m g ~ P ~ k g}^{-1}\right)\end{array}$} \\
\hline Fontes & $\mathbf{0}$ & $\mathbf{1 0 0}$ & $\mathbf{2 0 0}$ & $\mathbf{3 0 0}$ \\
SFT & $0,00 \mathrm{a}$ & $100,0 \mathrm{a}$ & $100,0 \mathrm{a}$ & $100,0 \mathrm{a}$ \\
FNB & $0,00 \mathrm{a}$ & $95,01 \mathrm{~b}$ & $91,01 \mathrm{c}$ & $94,01 \mathrm{~b}$ \\
FNT & $0,00 \mathrm{a}$ & $49,66 \mathrm{c}$ & $44,14 \mathrm{~d}$ & $38,16 \mathrm{~d}$ \\
FNR & $0,00 \mathrm{a}$ & $94,91 \mathrm{~b}$ & $97,50 \mathrm{~b}$ & $81,38 \mathrm{c}$ \\
\hline CV\% & \multicolumn{5}{c}{}
\end{tabular}

Médias seguidas por letras distintas dentro de cada dose diferem entre si pelo teste de Tukey a 5\% de probabilidade; C.V= Coeficiente de Variação; SFT= Superfosfato triplo; FNB= Fosfato Natural da Bahia; FNT $=$ Fosfato Natural do Tocantins; FNR= Fosfato Natural Reativo.

A análise de regressão para o índice de eficiência agronômica dos fosfatos naturais do presente estudo, em função das doses crescentes de fósforo, está representada na Figura 4. Demonstrado que os percentuais de eficiência das fontes FNB, FNT e FNR melhor se adequaram ao modelo quadrático de regressão, onde as máximas eficiências foram obtidas

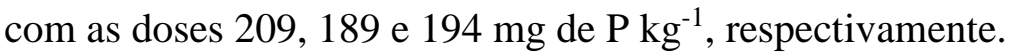

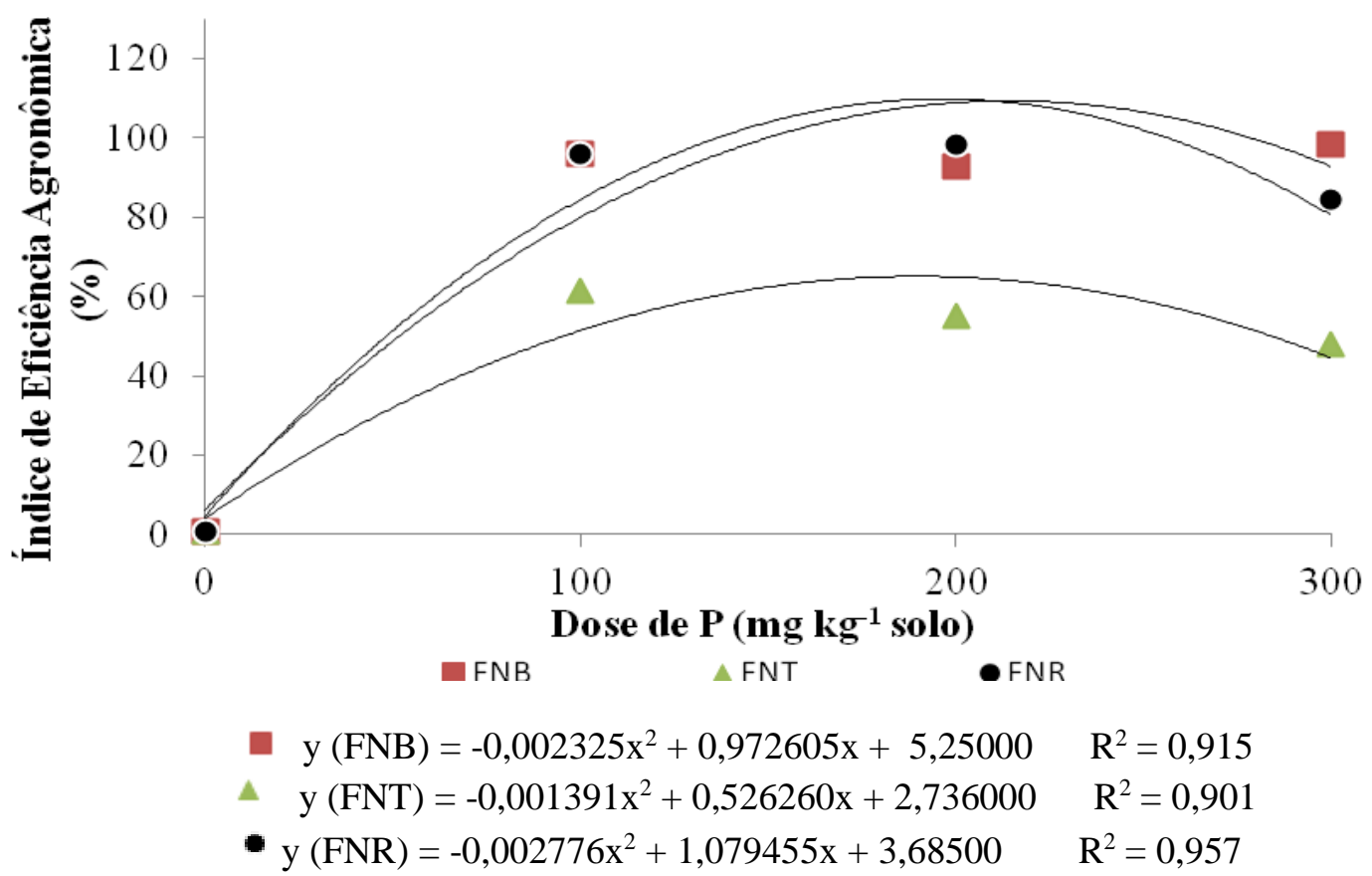

Figura 4. Análise de regressão do Índice de Eficiência Agronômica (\%) dos fosfatos naturais em função de doses crescentes de fósforo.

\section{CONCLUSÃO}

Para todas as variáveis, foi verificada interação significativa na relação fonte $\times$ dose. Verificou-se que a fonte Superfosfato triplo foi a que mais contribuiu para a absorção de Cultura Agronômica, Ilha Solteira, v.25, n.1, p.25-38, 2016 
fósforo do solo pelo sorgo e foi a fonte que apresentou maior Índice equivalente supertriplo e maior Índice de eficiência agronômica devido a sua alta solubilidade.

Os resultados são úteis para fazer uma recomendação de adubação fosfatada seguindo ordem decrescente de liberação de $\mathrm{P}$ para a planta: $\mathrm{ST}>\mathrm{FNB}>\mathrm{FNR}>\mathrm{FNT}$.

O fosfato natural do Tocantins é a fonte que menos contribuiu para o desenvolvimento das plantas de sorgo, o que indica que essa fonte possui baixo potencial agrícola. Dentre as fontes naturais, a FNB foi a única que apresentou média solubilidade, enquanto que as demais são classificadas como de baixa solubilidade.

\section{AGRADECIMENTOS}

A Fundação Cearense de Apoio ao Desenvolvimento Científico e Tecnológico FUNCAP, pela concessão da bolsa de mestrado, ao Professor Boanerges Freire de Aquino por toda orientação e ao Departamento de Ciências do Solo da Universidade Federal do Ceará.

\section{REFERÊNCIAS BIBLIOGRÁFICAS}

CHIEN, S. H.; MENON, R. G. Factors affecting the agronomic effectiveness of phosphate rock for direct application. Fertilizer Research, Dordrecht, v. 41, p.227-234, 1995.

CHIEN, S. H.; HAMMOND, L. L. A comparison of various laboratory methods for predicting the agronomic potential of phosphate rocks for direct application. Soil Science Society of America Journal, Madison, v. 42, p. 935-939, 1978.

CONAB. Companhia Nacional de Abastecimento. $\mathbf{7}^{\mathbf{0}}$ Levantamento da safra de grãos 2013-14.

http://www.conab.gov.br/OlalaCMS/uploads/arquivos/14_04_11_17_31_44_sorgomarco20 14.pdf. Acesso em: 29 dez. 2014.

CORRÊA, R. M.; NASCIMENTO, C. W. A.; SOUZA, S. K. S. S.; FREIRE, F. J.; SILVA, G. B. Gafsa rock phosphate and triple superphosphate for dry matter production and $P$ uptake by corn. Scientia Agrícola, Piracicaba, v. 62, n. 2, p.159-164, 2005.

COSTA, S. E. V. G. A.; NETO, A. E. F.; RESENDE, A. V.; SILVA, T. O.; SILVA, T. R. Crescimento e nutrição da Braquiária em função de fontes de fósforo. Ciência e Agrotecnologia, Lavras, v. 32, n. 5, p.1419-1427, 2008.

EMBRAPA. Centro Nacional de Pesquisa de Solos. Manual de Análises de Solo. $2^{\text {a }}$ ed., Rio de Janeiro: CNPS, 1997. 73 p.

EMBRAPA. Centro Nacional de Pesquisa de Solos. Sistema Brasileiro de Classificação de Solos. 2. ed., Rio de Janeiro: Embrapa Solos, 2006. 306 p.

Cultura Agronômica, Ilha Solteira, v.25, n.1, p.25-38, 2016 
FRANZINI, V.I.; MURAOKA, T.; MENDES, F.L. Ratio and rate effects of 32P-triple superphosphate and phosphate rock mixtures on corn growth. Scientia Agricola, Piracicaba, v. 66, p.71-76, 2009.

GILL, M. A.; SALIM, R. M.; ZIA, M. S. Maize growth and uptake of phosphate and copper at different ambient phosphate concentrations. Soil Science and Plant Nutrition, Tokyo, v. 8, n. 4, p.631-636, 1992.

GOEDERT, W. J.; LOBATO, E. Avaliação agronômica de fosfatos em solo de cerrado. Revista Brasileira de Ciência do Solo, Viçosa, v. 8, n. 1, p.97-102, 1984.

GOEDERT, W. J.; REIN, T. A.; SOUSA, D. M. G. Eficiência agronômica de fosfatos naturais, fosfatos parcialmente acidulados e termofosfatos em solo de cerrado. Pesquisa Agropecuária Brasileira, Brasília, v. 25, n. 4, p.521-530, 1990.

HOFMANN, C. R.; FAQUIN, V.; GUEDES, G. A. A.; EVAnGElistA, A. R. O nitrogênio e o fósforo no crescimento da braquiária e do colonião em amostras de um solo da região Noroeste do Paraná. Revista Brasileira de Ciência do Solo, Campinas, v. 19, n. 1, p.79-86, 1995.

KORNDÖRFER, G. H.; CABEZAS, W. A. L.; HOROWITZ, N. Eficiência agronômica de fosfatos naturais na cultura do milho. Scientia Agricola, Piracicaba, v. 56, n. 2, p.32-39, 1999.

LEITE, M. L. V. Crescimento vegetativo do sorgo Sudão (Sorghum sundanense (Piper) Stapf) em função da disponibilidade de água no solo e fontes de fósforo. 2006. 82 f. Dissertação (Mestrado em Zootecnia) - Universidade Federal da Paraíba, Areia, 2006.

LEÓN, L. A.; FENSTER, W. E.; HAMMOND; L. L. Agronomic potential of eleven phosphate rocks from Brazil, Colombia, Perú and Venezuela. Soil Science Society of America Journal, Madison, v. 50, n. 2, p.798-802, 1986.

LOPES, A. S. Manual de fertilidade do solo. Piracicaba: Fundação Cargill, 1989. 177 p.

MAGALHÃES, P. C.; DURÃES, F. O. M.; SCHAFFERT, R. E. Fisiologia da planta de sorgo. Sete Lagoas: Embrapa Milho e Sorgo, 2000. 46 p. (Embrapa Milho e Sorgo Circular Técnica, 3).

MALAVOLTA, E.; VITTI, G. C.; OLIVEIRA, S. A. Avaliação do estado nutricional das plantas: princípios e aplicações. 2. ed. Piracicaba: Potafos, 1997. 319 p.

MEURER, E. J. Fundamentos de química do solo. 3. ed. Porto Alegre: Evangraf, 2006. $285 \mathrm{p}$.

MOREIRA, A; MALAVOLTA. E; MORAES, L. A. C. Eficiência de fontes e doses de fósforo na alfafa e centrosema cultivadas em Latossolo Amarelo. Pesquisa Agropecuária Brasileira, Brasília, v. 37, n. 10, p.1459-1466, 2002.

NOVAIS, R. F.; SMITH, T. J. Fósforo em solos e planta em condições tropicais. Viçosa: Universidade Federal de Viçosa, 1999. 399 p.

Cultura Agronômica, Ilha Solteira, v.25, n.1, p.25-38, 2016 
OLIVEIRA, J. S.; FERREIRA, R. P.; CRUZ, C. D.; PEREIRA, A. V.; BOTREL, M. A.; PINHO, R. G. V.; RODRIGUES, J. A. S.; LOPES, F. C. F.; MIRANDA, J. E. C. Adaptabilidade e Estabilidade em Cultivares de Sorgo. Revista Brasileira de Zootecnia, Viçosa, v. 31, n. 2, p.883-889, 2002.

ROSAND, P. C.; SANTANA, M. B. Influência do tempo de contato e valor fertilizante de fontes fosfatadas em solos do sul da Bahia. Revista Theobroma, Ilhéus, v. 16, n. 1, p.1-15, 1986.

SANTOS, E. A.; KLIEMANN, H. J. Disponibilidade de Fósforo de Fosfatos naturais em Solos de Cerrado e sua Avaliação por Extratores Químicos. Pesquisa Agropecuária Tropical, Goiânia, v. 35, n. 3, p.139-146, 2005.

SARMENTO, P.; CORSI, M.; CAMPOS, F. P. Resposta da alfafa a fontes de fósforo associadas ao gesso e à calagem. Scientia Agrícola, Piracicaba, v. 58, n. 2, p.381-390, 2001 .

SEGATELLI, C. R. Produtividade da soja em semeadura direta com antecipação da adubação fosfatada e potássica na cultura de Eleusine coracana (L.) Gaertn. 2004. 58 f. Dissertação (Mestrado em Fitotecnia) - Escola Superior de Agricultura "Luiz de Queiroz", Universidade de São Paulo, Piracicaba, 2004.

SILVA, F. A. S.; AZEVEDO, C. A. V. Versão do programa computacional Assistat para o sistema operacional Windows. Revista Brasileira de Produtos Agroindustriais, Campina Grande, v. 4, n. 1, p.71-78, 2002.

TAIZ, L.; ZEIGER, E. Fisiologia vegetal. 3. ed. Porto Alegre: Artmed, 2004. 719 p.

VANCE, C. P.; STONE, C. U.; ALLAN, D. L. Phosphorus acquisition and use: critical adaptations by plants for securing a nonrenewable resource. New Phytologist, Lancaster, v. 157, n. 1, p.423-447, 2003. 